\title{
Variability, Optical-to-X-ray slope and accretion disc properties in a sample of Seyfert 1 AGN
}

\section{Ranjan Vasudevan*}

Department of Astronomy, University of Maryland, College Park, Maryland 20742, USA

E-mail: ranjan@astro.umd.edu

\section{Luigi Gallo}

Department of Astronomy \& Physics, Saint Mary's University, 923 Robie Street, B3H 3C3,

Halifax, Canada

E-mail: lgallo@ap.smu.ca

\section{Damien Robertson}

Department of Astronomy \& Physics, Saint Mary's University, 923 Robie Street, B3H 3C3,

Halifax, Canada

\section{Kendra Fulford}

Department of Astronomy \& Physics, Saint Mary's University, 923 Robie Street, B3H 3C3,

Halifax, Canada

\begin{abstract}
We present early results from an analysis of variability in the optical-to-X-ray spectral index, $\alpha_{\mathrm{OX}}$, in sample of 97 Seyfert 1-1.2 AGN. Simultaneous SEDs from XMM-Newton (PN and Optical Monitor instruments) are used to determine $\alpha_{\mathrm{OX}}$ and related SED parameters, using accretion disc model fits and accounting for intrinsic dust extinction. Our work supports the idea that the greater variability in $\alpha_{\mathrm{OX}}$ seen in Narrow-Line Seyfert 1 AGN (NLS1s) is driven primarily by changes in the corona, not the accretion disc. We confirm that NLS1s are accreting at high Eddington ratios, and also find higher peak temperatures in their accretion discs. We discuss the desirability of being able to constrain the inner radius of the accretion disc in a large sample of Broad- and Narrow-line Seyfert 1s, but this is currently difficult to do in large samples with the data available.
\end{abstract}

Narrow-Line Seyfert 1 Galaxies and their place in the Universe - NLS1, April 04-06, 2011

Milan Italy

\footnotetext{
*Speaker.
} 


\section{Introduction}

The standard paradigm for AGN includes a thermal accretion disc emitting in the optical-UV and a corona which up-scatters accretion disc photons to X-ray energies, producing power-law Xray emission. The spectral index connecting $2500 \AA$ and $2 \mathrm{keV}$ emission $\left(\alpha_{\mathrm{OX}}\right)$, sometimes referred to as the 'X-ray weakness', has been found to correlate with the $2500 \AA$ monochromatic UV luminosity in AGN $\left(L_{2500}\right)$, providing a probe of their accretion output across a broad wavelength range. Numerous determinations of this relation using various samples have been presented in the literature (notably [11],[10],[7],[6]). Some Narrow-Line Seyfert 1 (NLS1) AGN with spectral complexity have been found to deviate from this relation [4], especially exhibiting very low flux states in which the X-ray power law continuum severely reduces in flux. The wealth of simultaneous optical-UV and X-ray data from XMM-Newton provides an opportunity to understand the source and nature of this variability. In this proceedings, we present preliminary results from a systematic study of the variability in $\alpha_{\mathrm{OX}}$ in NLS1s in comparison to standard Seyfert 1s. A cosmology of $H_{0}=71$ and $\Omega_{M}=0.27$ is assumed throughout.

\section{The sample}

We are undertaking an analysis of simultaneous SEDs from XMM-Newton (using the PN and Optical Monitor instruments) for 97 low-absorption Seyfert 1-1.2 AGN, including a substantial fraction of NLS1 AGN. The objects are drawn from the samples of Grupe et al. [6], Boroson [2] and Sani et al. [9] along with any other individual sources in the $X M M$ archives which also have usable Optical Monitor (OM) data in at least one filter. Data are reduced according to the standard guidelines in the $X M M \mathrm{ABC}$ guide. We then impose an absorption cut, selecting only those with low X-ray absorption, requiring $N_{\mathrm{H}}<10^{21} \mathrm{~cm}^{-2}$. Low-absorption ensures a clean sample where the intrinsic emission can be more easily recovered. Multiple observations are available for 31 objects from the sample, allowing the effects of variability on determination of $\alpha_{\mathrm{OX}}$ and other SED parameters to be studied. The availability of optical-UV observations from XMM-OM allow the accretion disc properties to be probed, of course with the proviso that the host galaxy contamination should not be too severe. Virial ( $H \beta$ linewdith single-epoch) mass estimates were collected from the literature (from [9] and other sources) to allow fitting of accretion disc models, and ultimately to calculate the integrated bolometric luminosities $L_{\mathrm{bol}}$ and Eddington ratios $\left(\lambda_{\mathrm{Edd}}=L_{\mathrm{bol}} / L_{\mathrm{Edd}}\right.$, $\left.L_{\text {Edd }}=1.38 \times 10^{38}\left(M_{\mathrm{BH}} / M_{\text {sun }}\right) \mathrm{erg} / \mathrm{s}\right)$.

\section{Method}

The simultaneous XMM PN and OM data are fit with a simple multicolour blackbody accretion disc model (DISKPN) and broken power-law model combination (as done by Vasudevan et al. [12] for Swift-UVOT data), for all the datasets available. Dust extinction was incorporated using the XSPEC model ZDUST in the optical-UV regime along with the DISKPN model (the full model combination is ZDUST(DISKPN)+BKNPOWER). The DISKPN model takes 3 parameters: the maximal temperature in the disc $T_{\max }$, the inner radius $R_{\text {in }}$ and the normalisation $K=M_{\mathrm{BH}}^{2} \cos (\mathrm{i}) / \mathrm{D}_{\mathrm{L}}^{2} \beta^{4}$, where $M_{\mathrm{BH}}$ is the black hole mass, $i$ is the inclination angle of the disc to our line of sight, $D_{L}$ 


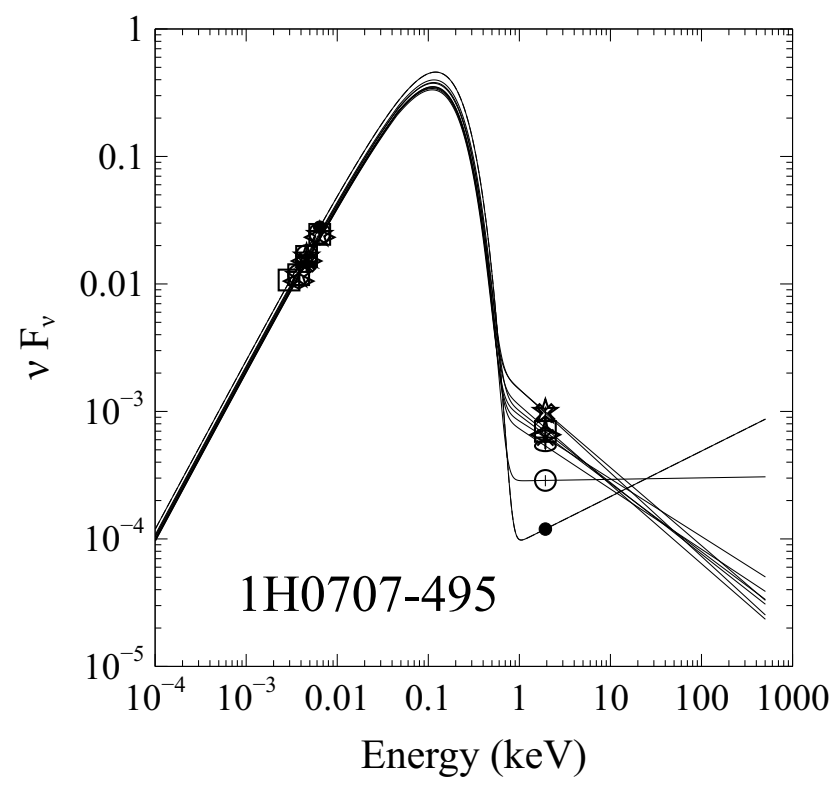

Figure 1: Multi-epoch SED for 1H0707-495 (all individual SEDs are constructed from simultaneous XMM-OM and PN data). Points with different symbols represent data taken at different epochs. Lines show the model fits to data points at a particular epoch.

is the luminosity distance to the source and $\beta$ is the spectral hardening parameter or "colour to effective-temperature ratio'. We freeze $R_{\text {in }}$ at $6 R_{\mathrm{g}}\left(R_{\mathrm{g}}=G M_{\mathrm{BH}} / c^{2}\right)$ and follow the assumptions discussed in [12] for $\beta$ and $i$. We reiterate the appropriateness of using an inclination angle of zero for these sources, since according to the unified paradigm for AGN [1] these type-1 Seyferts are likely to have the axes of their central engines at small angles to our line of sight. We use the virial mass estimates collected to constrain $K$, along with the redshift to provide the luminosity distance. We fix optical-UV intrinsic extinctions E(B-V) in the ZDUST model for individual objects when values are available in the literature ([13], [6]).

An example multi-epoch SED is shown for 1H0707-495 (Fig. 1) which has 8 XMM observations in the archives. For each object, we determine the dust-extinction-corrected $\alpha_{\mathrm{OX}}, 2500 \AA$ monochromatic luminosity $L_{2500}, L_{\mathrm{bol}}$ and $\lambda_{\text {Edd }}$ from the model fits. Our results are compared with the existing determinations of the $\alpha_{\mathrm{OX}}-L_{2500}$ relation in Fig. 2, where filled circles represent the results from extinction corrected model fits. Values of $\alpha_{\mathrm{OX}}$ and $L_{2500}$ are also calculated from the raw data (empty circles in Fig.2), as is standard in the works on larger samples (e.g. [11] and subsequent papers). Visual comparison of this plot with those in previous works reveals a somewhat tighter relation between $\alpha_{\mathrm{OX}}$ and $L_{2500}$, which could be in part due to to the use of simultaneous data.

We present our preliminary results on the variability of $\alpha_{\mathrm{OX}}$ in two ways. Firstly, we show how the values of $\alpha_{\mathrm{OX}}$ vary within one particular source, 1H 0707-495, in Fig. 3. Secondly, we present the variation in the whole sample in Fig. 4. The results are presented as $\Delta \alpha_{\mathrm{OX}}$, the deviation of the model-determined $\alpha_{\mathrm{OX}}$ from the best-fit relation between $\alpha_{\mathrm{OX}}$ and $L_{2500}$ as calculated from the raw 


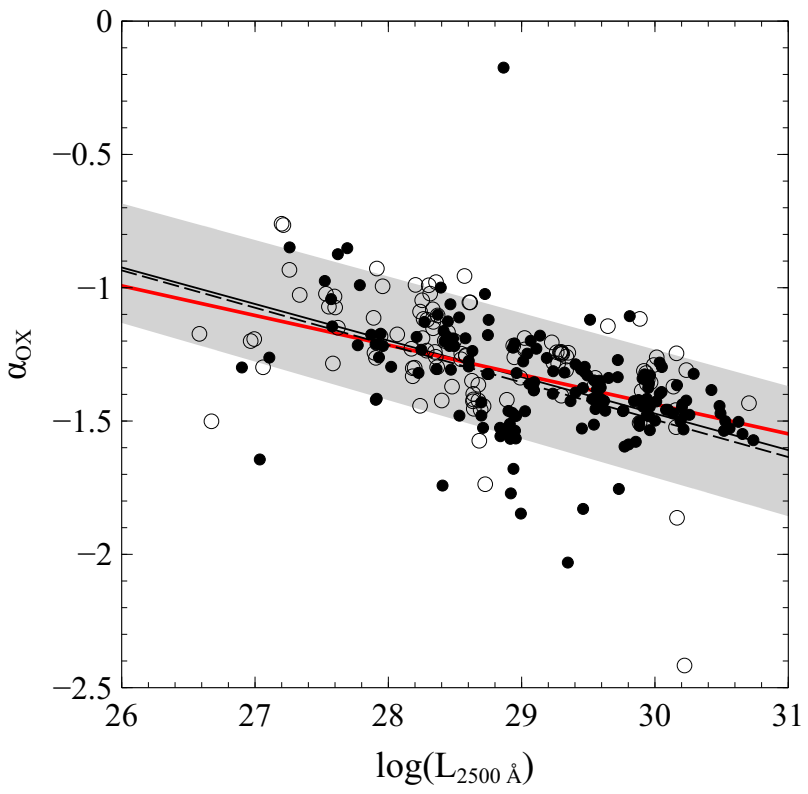

Figure 2: $\alpha_{\mathrm{OX}}$ vs luminosity at $2500 \AA$. Empty circles represent values determined from the raw data without any model fitting or removal of intrinsic extinction. Filled circles represent values from model fits. The solid black line depicts the Steffen et al. (2006) [10] relation (with grey showing the spread), the dashed line the Just et al. (2007) [7] relation, and finally the red line shows the best fit between the raw-data determinations of $\alpha_{\mathrm{OX}}$ and $L_{2500}$.

data (the red line in Fig. 2) where positive $\Delta \alpha_{\mathrm{OX}}$ represents X-ray weakness. In the former plot (Fig. 3), we plot $\Delta \alpha_{\mathrm{OX}}$ against the maximal disk temperature from the DISKPN model fit; there are hints of a correlation, but this appears to be the only object in our sample which a) shows such a correlation, and b) has such a large number of multi-epoch observations to discern such a relation. In the latter plot (Fig. 4), we plot $\Delta \alpha_{\mathrm{OX}}$ against the traditional discriminant between narrow lineand broad line-Seyfert $1 \mathrm{~s}$, the $H \beta$ full-width half-maximum (FWHM). Multiple observations for individual objects are depicted using coloured symbols and connector lines linking all the observations for a particular source. It can be seen clearly that the extinction-corrected $\alpha_{\mathrm{OX}}$ varies far more substantially in NLS1s (FWHM $<2000 \mathrm{kms}^{-1}$ ) compared to their standard Seyfert 1 counterparts. We also point out, as previously noted in [4] that NLS1s tend to dip into an X-ray weak state more frequently.

\section{Accretion properties}

\subsection{Maximum temperature in the accretion disc}

Fig. 5 shows the maximum temperature in the accretion disc determined from the DISKPN fits to the optical-UV data, against the FWHM of the $H \beta$ linewidth. As before, multiple colours illustrate multi-epoch observations of individual objects. This characteristic temperature in the 


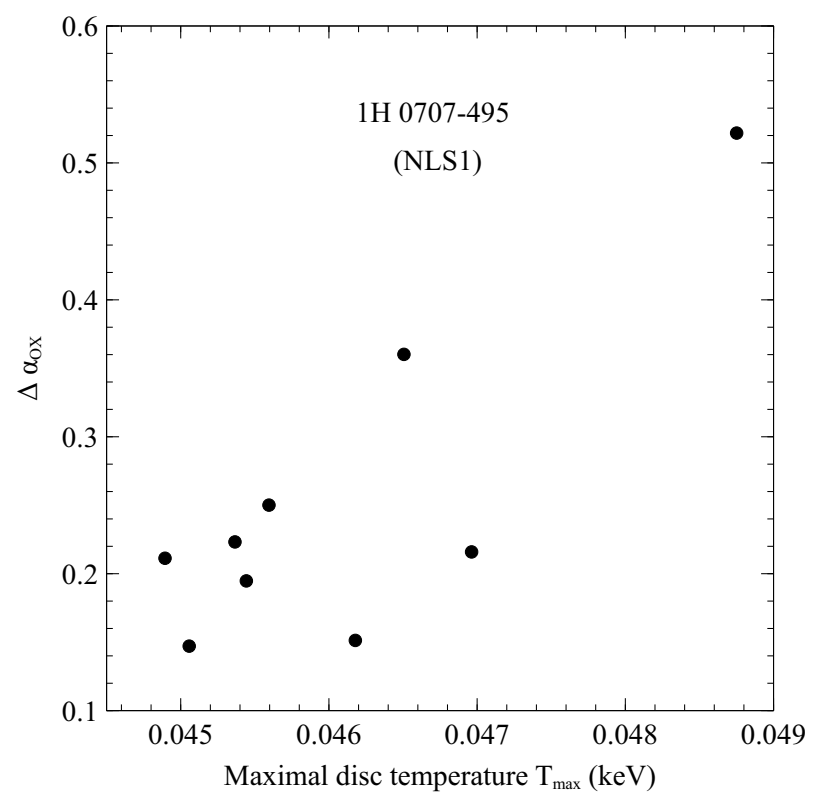

Figure 3: $\Delta \alpha_{\mathrm{OX}}$ (with respect to the best fit from this work, the red line in Fig. 2) against peak accretion disc temperature $T_{\max }$ for $1 \mathrm{H} 0707-495$.

accretion disc appears to be systematically higher in NLS1s, as expected for their typically smaller black hole masses. We also see that the accretion disc temperature remains largely constant across epochs in individual objects, suggesting that the overwhelming source of the variability in $\alpha_{\mathrm{OX}}$ is the X-ray variability (see Fig. 1).

\subsection{Eddington ratio}

We also confirm that NLS1s have higher Eddington-scaled accretion rates in this sample, here using simultaneous SEDs and direct SED integration to determine the bolometric luminosities and Eddington ratios (Fig. 6, key as for Fig. 5). The distribution of Eddington ratios appears qualitatively similar to that for the maximal disc temperatures presented in Fig. 5, but we note the presence of a few objects with unphysically high Eddington ratios. Those with the highest Eddington ratios are IRAS 13224+3809, PHL 1092, Mrk 493, which all have $\lambda_{\text {Edd }}>20$. The object with the highest Eddington ratio, IRAS $13224+3809$, also has the second smallest $\mathrm{BH}$ mass in the entire sample, and PHL 1092 and Mrk 493 also have small black hole masses $\left(\log \left(\mathrm{M}_{\mathrm{BH}} / \mathrm{M}_{\text {sun }}\right)<6.6\right)$. These large Eddington ratios could be due to either the small black hole mass, or too large an extrapolation of the unobserved big-blue-bump in the far-UV using the DISKPN model. Since the black hole masses used are calculated from $\mathrm{H} \beta$ linewidths themselves, it is likely that there are more complex dependences at at work in Fig. 6 which deserve further investigation. 


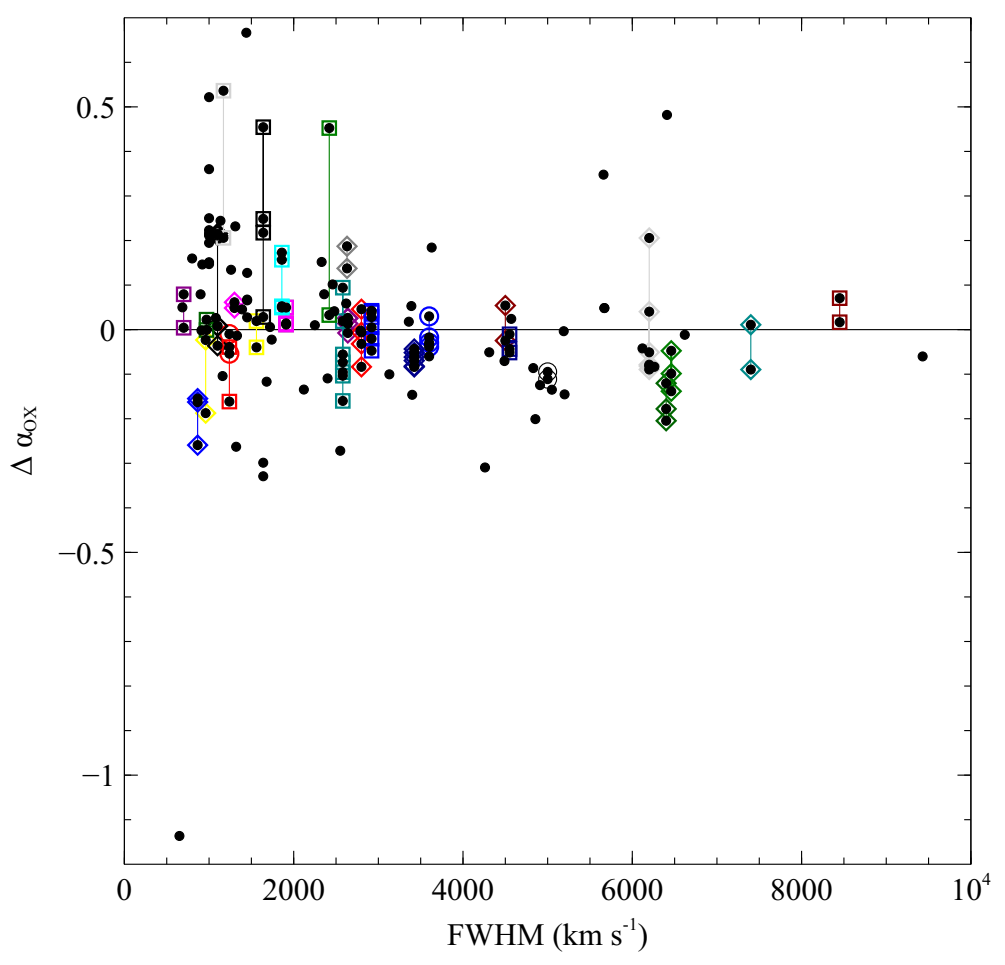

Figure 4: $\Delta \alpha_{\mathrm{OX}}$ against $H \beta$ FWHM $\left(\mathrm{kms}^{-1}\right)$ for the whole sample. Multiple observations of the same object are shown with colour symbols enclosing each point, with the multiple values for individual objects joined by a connector line. A clear trend towards bigger multi-epoch variations in $\alpha_{\mathrm{OX}}$ at low FWHM is seen.

\section{Summary and Future Work}

We present early results from an analysis of variability in $\alpha_{\mathrm{OX}}$ in a sample of Seyfert 1-1.2s using simultaneous SEDs from XMM-Newton, forming a complement to the work done by Grupe et al. [6] using simultaneous SEDs from Swift. Our work seems to suggest that $\alpha_{\mathrm{OX}}$ is intrinsically more variable in NLS1s, with the X-rays driving the variability, reinforcing this conclusion of Grupe et al. [6] with an extended sample. This work avoids uncertainties due to epoch-to-epoch variability in SEDs by using simultaneous optical-UV and X-ray data from XMM Newton's PN and OM instruments. Our findings so far would suggest that the accretion disc remains relatively constant while the variations in the corona must be responsible for the broad-band SED variation probed by $\alpha_{\mathrm{OX}}$.

We also confirm previous trends for NLS1s to have higher Eddington ratios, now using simultaneous data to determine the bolometric luminosities. A trend for NLS1s to also have higher accretion disc peak temperatures closely mirrors this, as expected from the dependence of disc temperature on accretion rate in standard accretion discs, but we caution about the presence of some highly super-Eddington sources and find that the virial mass estimates employed may need closer 


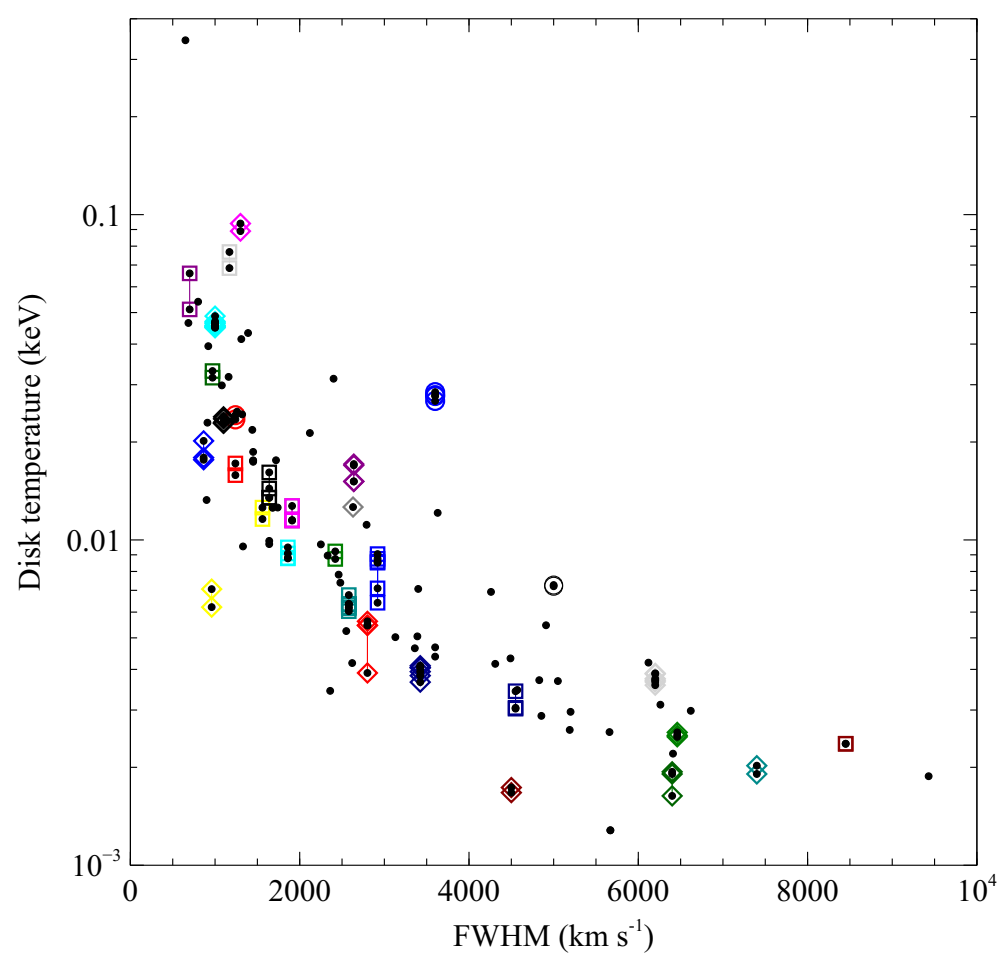

Figure 5: Maximum temperature in the accretion disc, $T_{\max }$, against $H \beta \mathrm{FWHM}\left(\mathrm{kms}^{-1}\right)$ for the whole sample. Key as in Fig. 2.

inspection. If the masses are too small, this could produce the unphysically super-Eddington ratios seen. Once these issues are addressed, we also aim to present $2-10 \mathrm{keV}$ bolometric corrections for this sample.

It would also be instructive to determine if there is any variability in the inner disc radius, between epochs in individual objects and across the sample as a whole. At present, the lack of data in the far-UV makes it difficult to constrain the inner disc radius from simple DISKPN fits. If the turnover in the accretion disc spectrum is observed, for example, then a more useful constraint on the inner disc could be obtained. Determining the inner disc radius would be highly desirable, as it can also help to answer questions about the link between the host galaxy type, the fuelling process and the NLS1 nature of an individual source. For example, Orban de Xivry et al. [8] and the presentation from R. Davies at this meeting [3] suggest that the high Eddington ratios of NLS1s could reflect a high spin (hence smaller inner accretion disc radius), possibly the result of prolonged accretion from within a 'pseudo-bulge' type host galaxy characteristic of secularlyevolved galaxies. Additionally, measures of the inner disc radius would provide a useful perspective on whether strong light bending is at work in NLS1s, to account for their rapid variability and spectral features in the X-ray weak state [5]. Constructing a database of disc inner radii/black hole spins for Seyfert 1 AGN would help to solve some of these puzzles. 


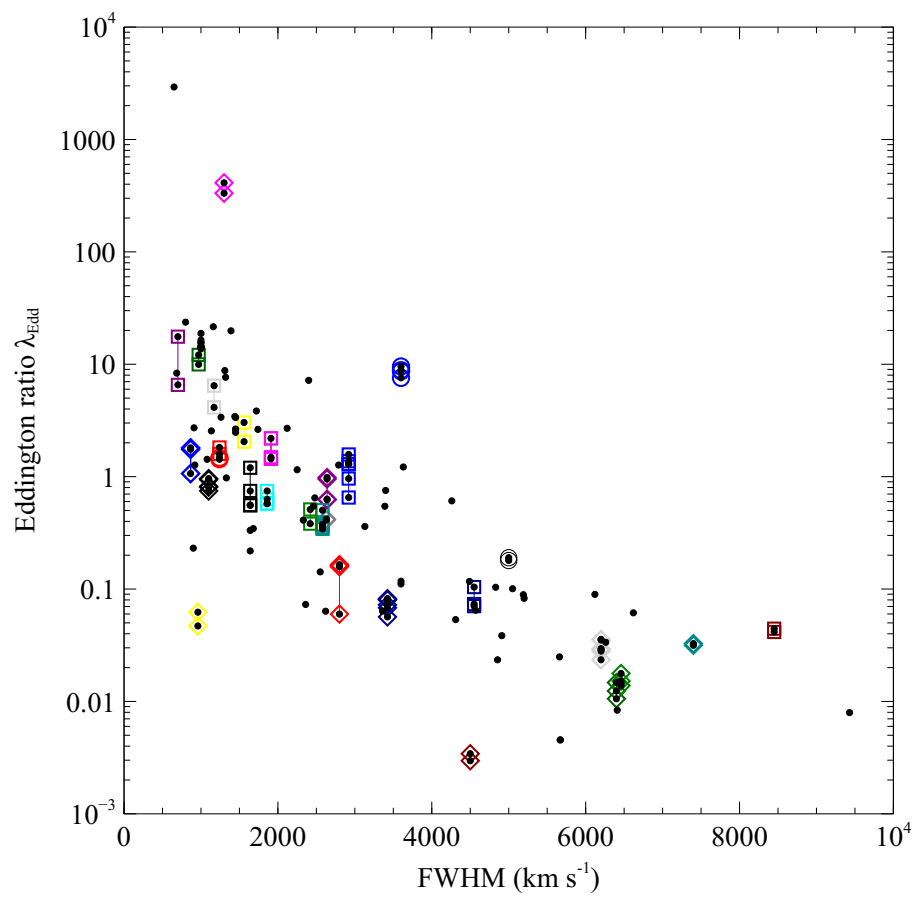

Figure 6: Eddington ratio $\lambda_{\mathrm{Edd}}=L_{\mathrm{bol}} / L_{\mathrm{Edd}}$ against $H \beta \mathrm{FWHM}\left(\mathrm{kms}^{-1}\right)$ for the whole sample. Key as in Fig. 2.

\section{Acknowledgments}

The author thanks L. Gallo for the invitation to collaborate on this project.

\section{References}

[1] R. Antonucci: Unified models for active galactic nuclei and quasars, 1993, ARAA, 31, 473.

[2] T. A. Boroson: Black Hole Mass and Eddington Ratio as Drivers for the Observable Properties of Radio-loud and Radio-quiet QSOs, 2002, ApJ, 565, 78.

[3] R. Davies: Cosmic Evolution of NLSI and the Growth of their Black Holes. In: Proceedings of the Workshop Narrow-Line Seyfert 1 Galaxies and Their Place in the Universe, PoS (NLS1) 036 (2011).

[4] L. C. Gallo: Investigating the nature of narrow-line Seyfert 1 galaxies with high-energy spectral complexity, 2006, MNRAS, 368, 479.

[5] L. C. Gallo: The X-ray weak state of Narrow-Line Seyfert 1 galaxies. In: Proceedings of the Workshop Narrow-Line Seyfert 1 Galaxies and Their Place in the Universe, PoS (NLS1) 017 (2011).

[6] D. Grupe et al. The Simultaneous Optical-to-X-Ray Spectral Energy Distribution of Soft X-Ray Selected Active Galactic Nuclei Observed by Swift, 2010, ApJS, 187, 64.

[7] D. W. Just et al. The X-Ray Properties of the Most Luminous Quasars from the Sloan Digital Sky Survey, 2007, ApJ, 665, 1004. 
[8] G. Orban de Xivry et al. The Role of Secular Evolution in the Black Hole Growth of Narrow-Line Seyfert 1 Galaxies, 2011, submitted to MNRAS (arXiv:1104.5023)

[9] E. Sani et al. Enhanced star formation in narrow-line Seyfert 1 active galactic nuclei revealed by Spitzer, 2010, MNRAS, 403, 1246.

[10] A. T. Steffen et al. The X-Ray-to-Optical Properties of Optically Selected Active Galaxies over Wide Luminosity and Redshift Ranges, 2005, AJ, 131, 2826.

[11] I. V. Strateva et al. Soft X-Ray and Ultraviolet Emission Relations in Optically Selected AGN Samples, 2005, AJ, 130, 387.

[12] R. V. Vasudevan et al. Optical-to-X-ray emission in low-absorption AGN: results from the Swift-BAT 9-month catalogue, 2009, MNRAS, 399, 1553.

[13] L. M. Winter et al. Optical Spectral Properties of Swift Burst Alert Telescope Hard X-Ray-Selected Active Galactic Nuclei Sources, 2010, ApJ, 710, 503. 